\title{
Remote associations and recognition memory for serial position
}

\author{
G. J. JOHNSON, DON JAMIESON, and CLYDE CURRY \\ University of British Columbia, Vancouver, British Columbia, Canada V6T 1W5
}

\begin{abstract}
After having received training on a serial list, subjects were shown a series of number-syllable pairs that represented correct or remote positional associations assumed to be formed during serial learning. The subjects indicated for each pair whether or not the number corresponded to the serial position of the syllable. The frequency with which a given association was erroneously recognized as "correct" was a negative exponential function of degree of positional remoteness. The results indicate that the "gradient of remote associations" is not an artifact of differential availability of serial-list items.
\end{abstract}

Traditionally the concept of remote associations has been linked to Ebbinghaus' "sequential association" analysis of serial learning. According to this analysis, the consistent temporal succession in which the items in a serial list are presented gives rise not only to direct (i.e., adjacent-item) associations but to remote associations as well. In the context of Ebbinghaus' view of serial learning, "remote association" refers to a connection between a given pair of items occupying nonadjacent positions in a serial list. The strength of a remote association is assumed to be an inverse function of its degree of remoteness, that is, the number of items that separate a given pair of items in the list. Until the last decade or so, remote associations provided a widely recognized basis for accounting for a variety of serial-learning phenomena. However, in view of several different lines of recent research (see Ebenholtz, 1972 or Young, 1968 for a review of this evidence), the traditional version of the remote associations theory must be regarded as inadequate. The difficulties with it appear to reflect the inaccuracy of two assumptions: (1) that the "effective stimuli" for remote associations are the serial-list items themselves, and (2) that the development of remote associations of differing strength depends upon a consistent temporal succession of item presentation.

Johnson (1975a) has proposed a modified version of the remote associations concept that appears to be consistent with results that demonstrate the inadequacies of the original version. This modification involves a reconception of remote associations in terms of a positional association model of serial learning. The associative process that is viewed as being essential to serial learning is the formation of associations, not among the individual items themselves, but among the items and cues that represent the positions of the items along an ordinal continuum. Generalization of response tendencies across

This research was supported by a grant from the National Research Council of Canada, APA 02796. Don Jamieson is now at Carleton University. This paper is sponsored by Roderick Wong, who takes full editorial responsibility for it. positional cues is assumed to result in the development of remote positional associations. This means that each position, as a "stimulus," comes to be associated to a varying degree with each of the items in a list. Accordingly, from this view a "remote association" refers not to a connection between a given pair of serial-list items, but to a connection between a given positional cue and an item that occupies some other position in a serial list. The strength of a remote association is considered to be inversely related to the positional displacement of a given one of the items relative to a given ordinal position. That is, if the direct associations relevant to serial learning are represented as 1-A, 2-B, 3-C, 4-D, etc., this analysis implies that the strength of the remote associations $1-B$ and $2-A$ is greater than that of $1-C$ and $3-A$ which, in turn, is greater than that of $1-D$ and $4-A$, and so forth.

One of the issues to which the positional model may be applied concerns the characteristic "gradient of remote associations" that is reflected (a) in the pattern of intralist intrusion errors that subjects make as they are learning a serial list and (b) in the results of an association task that is imposed upon the subjects after they have learned a serial list. For the analysis of intrusion errors the misplaced responses are classified as to their degree of displacement relative to their correct locations in the list. This analysis shows that frequency of intrusions is a negatively accelerated inverse function of degree of displacement (e.g., Slamecka, 1964). A similar pattern of results is obtained when the "association method" is used. After the subjects have learned a serial list, they are presented with each of the serial-list items (singly, in a random order) and asked to respond in each instance with the first of the other items that comes to mind. As the degree of positional remoteness between a given pair of items increases, the likelihood that, in the association task, one of them will elicit the other decreases exponentially (e.g., McGeoch, 1936).

Johnson (1975a) has shown how the concept of remote positional associations can be used to account for these findings. However, there is a particular problem 
that must be dealt with before this interpretation of the remote associations gradient can be accepted. In a critique of the traditional concepts, Slamecka (1964) pointed out that such gradients may be an artifact of differential response availability of the items in a serial list. It is likely that those items more frequently given as correct responses during serial learning (i.e., the ones in the beginning and in the end portions of the list) have a higher degree of availability than do the items in the middle portion of the list. If this is so, then the items at the ends of the serial list may be more frequently emitted during serial learning or during an association task than are the items in the middle of the list. Slamecka suggests that this factor, in and of itself, might be sufficient to produce the essential characteristics of the gradient. Although this criticism was directed toward the traditional remote associations interpretation of the gradient, it is equally appropriate to the one that is implied by defining remote associations in terms of position-item relations. That is, it is possible that the shape of the gradient has little to do with either itemitem associations or position-item associations.

The present study was conducted in an attempt to eliminate any influence that differential availability of serial-list items might have on the shape of the remote associations gradient. The subjects were first given training on a serial list and then administered a positionrecognition task. For this task they were asked to respond simply with "yes" or "no" as a means of indicating whether or not a particular position-item association was "correct" with respect to the serial list. Since the subjects were not required to emit the seriallist items as responses, differences in performance as a function of the remoteness of the position-item associations cannot be attributed to the response availability factor.

\section{METHOD}

\section{Procedure}

Three 10-item serial lists were constructed from a set of 10 high-frequency CVC nonsense syllables. Each of the items occupied a different serial position in each list, and no two items occurred in adjacent positions in more than one of the lists. The items were presented by tape recorder at a 3-sec rate. As each item was presented aurally, the experimenter showed to the subjects a large card upon which was printed the appropriate item. After each presentation of the list, the subjects attempted to write down the items in serial order on a response sheet that contained 10 lines. They were given $30 \mathrm{sec}$ to record their responses, and then they were asked to turn to the next page in the response booklet. A series of three study-test trials was conducted in this manner.

For the position-recognition task the subjects were presented (again by tape recorder, at a 3-sec rate) a series of number-syllable pairs. Each of these items represented a combination of one of the numbers, $1-10$, and one of the 10 syllables from the serial list. The subjects' task was to indicate in each instance whether or not the particular syllable was the one that, in the serial list, had occupied the position designated by the given number. Each of the number-syllable pairs corresponded to one of the 90 remote associations or one of the 10 direct associations relevant to the serial list. The "correct" associations were included to discourage subjects from developing a set to respond "no" to all of the items. One hundred and twenty number-syllable pairs were presented in 12 blocks of 10 items each. Six of the blocks each contained seven items that represented remote associations and three items that represented direct associations. The remaining six blocks each contained eight remote-association and two direct-association items. Within each block of 10 items, a given number or a given syllable appeared only once. With the exception of this restriction and the one determining the number of remote and direct associations within a block, the order in which the 120 number-syllable pairs was presented was random.

For each of the items the subject indicated his response by underlining "yes" or "no" at the appropriate location on a response sheet. Each of two response sheets contained six blocks of 10 "yes-no" items arranged vertically on a page. In order to prevent the subjects from "losing their place," the items appeared on a page with single spacing among individual items within a block and double spacing between successive blocks. Further, in the aural presentation of the number-syllable pairs. a 3-sec break occurred between each block of 10 items.

\section{Subjects}

Two hundred and twenty-seven introductory psychology students participated in the study. The subjects were run in three groups of approximately equal size, with a different one of the serial lists being used for each of the groups.

\section{RESULTS AND DISCUSSION}

The frequency with which the subjects responded incorrectly (by indicating "yes") was tabulated for each of the 90 remote associations relevant to the serial list. The total number of errors on the position-recognition task was 3,637 . Three different analyses were applied to these data. First, the errors were classified on the basis of the ordinal positions of the stimulus terms of the number-syllable pairs. The percentage of errors that occurred with each of the numbers $1-10$ as stimulus is shown in Figure 1. Second, percentage of errors as a function of the serial-list positions of the response terms was calculated. The result of this analysis is also shown in Figure 1.

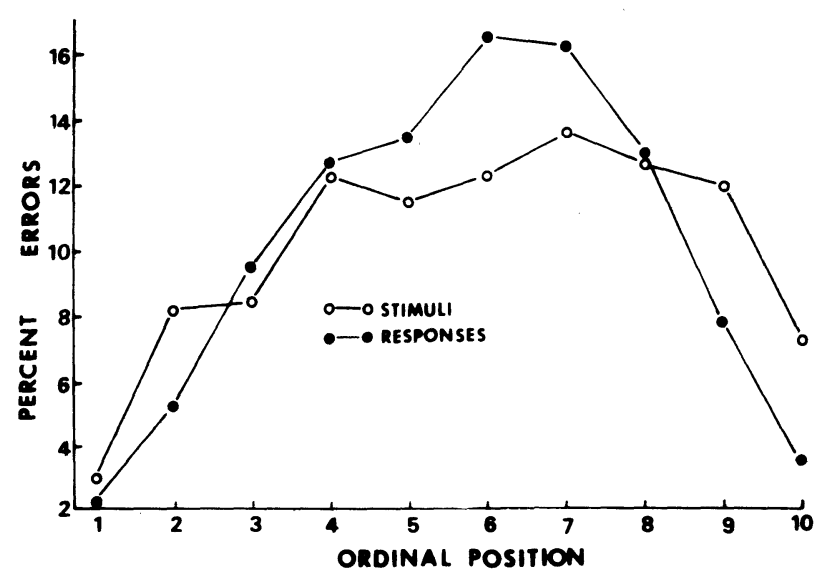

Figure 1. Percentage recognition errors on the numbersyllable pairs as a function of ordinal position of the stimuli and ordinal position of the responses. 


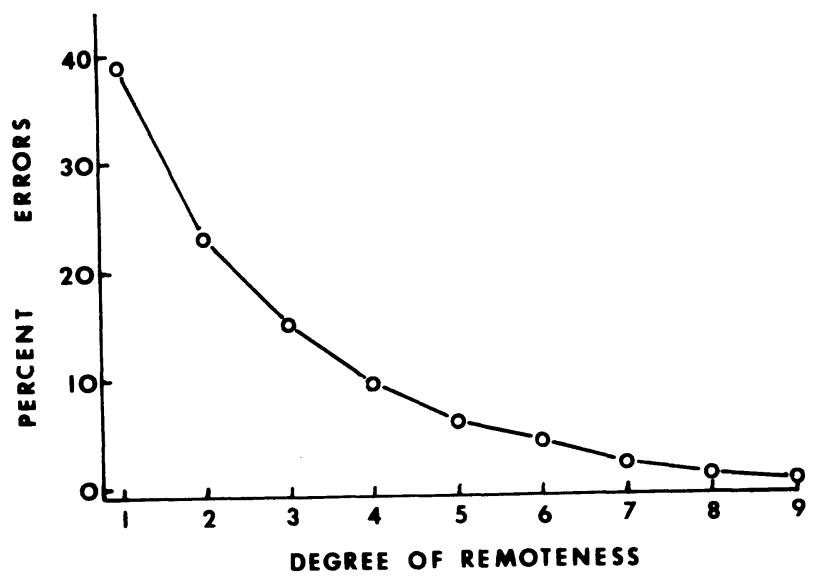

Figure 2. Percentage recognition errors as a function of degree of positional remoteness of the number-syllable pairs.

The most notable feature of the results that appear in Figure 1 is that both curves approximate the ordinal position curve that is characteristic of serial acquisition data. In this respect, the results are in agreement with those of Schulz (1955). After having received training on a serial list, the subjects in Schulz's study were presented with each of the items (singly, in random order) and asked to recall the position that the item had occupied in the serial list. The relative difficulty of the items in the position-naming task corresponded very closely to their relative difficulty in serial learning. These results were viewed as support for the assumption that ordinal position effects in serial learning reflect the differential strength of generalized positional associations.

The third analysis that was applied to the present data concerns the relative level of difficulty of the number-syllable pairs as a function of degree of remoteness. Presented in Figure 2 are the percentages of errors on the position-recognition task that corresponded to serial-list associations of one through nine degrees of remoteness. This gradient is biased, of course, by the differential opportunities that the subjects had for making errors that correspond to the various degree of remoteness. The ratio of opportunities for errors representing associations of one through nine degrees is $9: 8: 7: 6: 5: 4: 3: 2: 1$. Thus on the basis of this consideration alone, the percentage of first-degree recognition errors, for example, would be expected to be $9 / 45$, or $20 \%$. The percentage of second-degree errors would be expected to be $8 / 45$, or $18 \%$, and so forth. The Kolmogorov-Smirnoff test was used to assess the significance of the difference between the observed gradient and the one that would be expected on the basis of differential opportunity for occurrence. The difference was significant beyond the .01 level.

Differences in recognition performance are clearly uncontaminated by the effects of item availability. Yet, the results in Figure 2 show the essential characteristics of the typical remote associations gradient. The subjects inaccurately identified a given number-syllable pair as "correct" most often when the serial position of the syllable was adjacent to the one indicated by the position cue. As the ordinal distance between a position cue and the serial-list location of a syllable increased, the frequency of recognition errors decreased exponentially. Frequency of errors on the recognition task may be taken as a measure of the strength of associations established during serial leaning. Accordingly, the results may be interpreted as support for the view that serial learning entails the formation of position-item associations whose strength varies inversely with degree of remoteness.

There is a growing amount of evidence that intralist generalization of positional associations may underlie a variety of serial learning phenomena. For example, Fishbein, Shackney, and Sinclair (1965) and Schulz (1955) have shown that the relative difficulty of seriallist items as a function of ordinal position may be interpreted by these terms. The concept of generalized positional associations was used by Dey (1970) to account for the pattern of intra- and interlist intrusions that subjects make as they are learning two serial lists concurrently. Johnson (1975b) indicated the manner in which remote positional associations may be related to the transfer effects that are obtained with the serialserial "derived-list" paradigm. In sum, it appears that the usefulness of the remote associations concept does not depend upon the validity of Ebbinghaus' theory of serial learning, to which it has traditionally been linked. It might be worthwhile to explore further the implications of the concept as it is construed within the framework of a positional association analysis of serial learning.

\section{REFERENCES}

DEY, M. K. Generalization of position association in rote serial learning. American Journal of Psychology, 1970, 83, 248-255.

Ebenholtz, S. M. Serial learning and dimensional organization. In G. H. Bower (Ed.), The psychology of learning and motivation: Advances in research and theory (Vol. 5). New York: Academic Press. 1972.

Fishbein, H. D., Shackney, C., \& Sinclair, D. A stimulus generalization explanation of the serial position effect. Psychonomic Science, 1965, 3, 563-564.

Johnson, G. J. Positional mediation of intraserial associations. American Journal of Psychology, 1975, 88, 49-63. (a)

Johnson, G. J. Transfer of serial learning as a function of interlist positional relations. Canadian Journal of Psychology. 1975. 29. 183-194. (b)

McGeосн. J. A. The duration and extent of intra-serial associations and recall. American Journal of Psychology, 1936. 48. $221-245$.

Schulz. R. W. Generalization of serial position in rote serial learning. Journal of Experimental Psychology. 1955, 49. 267-272.

Slamecka, N. J. An inquiry into the doctrine of remote associations. Psychological Review. 1964, 71, 61-76.

Young. R. K. Serial learning. In T. R. Dixon \& D. L. Horton (Eds.). Verbal behavior and general behavior theory. Englewood Cliffs, N.J: Prentice-Hall. 1968.

(Received for publication January 30, 1976.) 